\title{
TRANSPORT PROPERTIES AND CURRENT FLOW PATTERNS IN HOMOGENEOUS STRONGLY ANISOTROPIC MATERIALS
}

\author{
Richard D. Chippendale, Chi Zhang, Igor O. Golosnoy, Paul L. Lewin and Jan K. Sykulski \\ School of Electronics and Computer Science, University of Southampton, Southampton, SO17 1BJ, UK \\ e-mail: rc805@ecs.soton.ac.uk, ig@ecs.soton.ac.uk
}

\begin{abstract}
A finite element model has been used to study potential distribution and current flow paths in highly anisotropic composite materials. Unexpected results are obtained, which are found consistent with minimising of Joule heat release in the material.
\end{abstract}

\section{INTRODUCTION}

Carbon fibre composites (CFC) consist of two components with very different properties. These components are combined together to obtain a material suitable for a wide range of applications [1]. Normally CFCs contain several laminates arranged in plain layers. Each laminate has continuous carbon fibres aligned along one direction. The large difference in conductivity of epoxy and carbon fibre results in highly anisotropic characteristics of individual laminates [2, 3]. Assembled into the panel, the laminates provide complex conductive paths for electric current and heat flows within the layered structure. There has been very little work into investigating the transport properties of commercial CFCs panels [4] with most studies focused on unidirectional laminates [2, 3, 5, 6]. Previous studies looked at averaged transport properties, whereas in this paper we predict the potential distribution and electrical current flow in CFC panels using a homogenous anisotropic material model. (The results can also be used to analyse heat flow patterns.)

\section{PROBLEM FORMULATION}

\section{A. Material properties and layout}

As a first approximation, it is assumed that the CFC's conductivity is represented by a tensor with two different electrical conductivities $\sigma_{11}$ and $\sigma_{22}=\sigma_{33}$, with the principle direction " 1 " coinciding with the direction of carbon fibres, and the directions " 2 " and " 3 " assumed normal to the fibre direction. It has been observed that $\sigma_{11}$ is $2-5$ orders of magnitude larger than the conductivities $\sigma_{22}=\sigma_{33}[2,3,5,6]$. For our modelling purposes, the CFC material is assumed to have $\sigma_{11}=2000 \mathrm{~S} / \mathrm{m}$ and $\sigma_{22}=\sigma_{33}=20 \mathrm{~S} / \mathrm{m}$. The model is designed using two square layers of CFC laminates stacked on top of each other. The analysis is done for the panel made of two laminates with the principle direction " 1 " rotated by $90^{\circ}$ in the bottom layer, see Fig. 1. Two circular electrodes are placed above and below the centre of the CFC stack, with a potential difference $1 \mathrm{~V}$ applied across the electrodes. The dimensions and overall diagram of the model are shown in Fig. 1.

\section{B. Governing equations and boundary conditions}

The solution for the steady state current flow problem in terms of the electric current density $\mathbf{J}$ and the electric field $\mathbf{E}$ is given by (1), which in turn - together with Ohm's law - results in formulation (2) for the electric potential $\varphi$ :

$$
\begin{gathered}
\nabla \cdot \mathbf{J}=0, \mathbf{E}=-\nabla \varphi, \mathbf{J}=\sigma \mathbf{E} \\
\nabla \cdot \sigma \nabla \varphi=0
\end{gathered}
$$

The application of the Dirichlet's principle to (1)-(2) is equivalent to seeking the minimum of the Joule heat problem:

$$
\varphi \rightarrow \min \left(\iiint_{\text {volume }} \mathbf{J} \cdot \mathbf{E} d v\right)
$$

The external boundary conditions are provided by electrical insulation on all surfaces except where the electrodes are placed. The potential for the top electrode is assumed to be $\varphi_{\text {top }}=1 \mathrm{~V}$, and the bottom electrode is grounded.

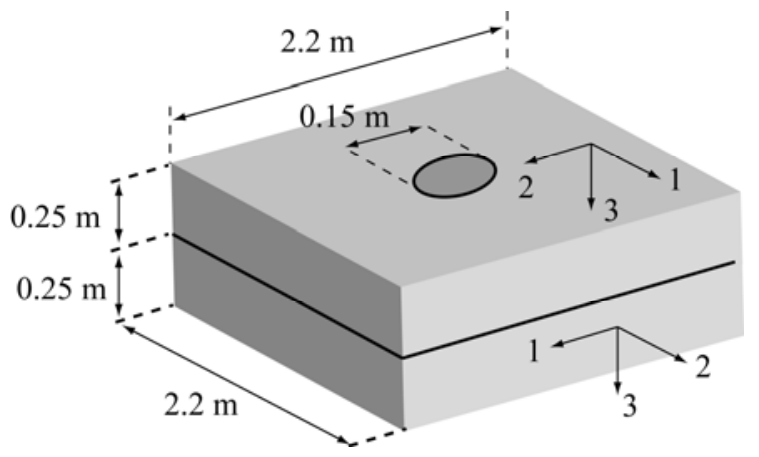

Fig.1. Schematic diagram of the CFC Stack model

Commercial finite element software COMSOL [7] was used for the computation. Cubic elements were used with 110 nodes uniformly spaced along the in-plane directions and 26 nodes through the thickness. It was found that smooth equipotential surfaces could be obtained by linear shape functions. Due to a complex shape of the equipotential surfaces the application of quadratic or higher order shape functions results in a wobbling of the surfaces and a drop in the model accuracy.

\section{RESULTS}

The equipotential surfaces within $\pm 1 \%$ of the mid potential $0.5 \varphi_{\text {top }}$ have a twist, as the potential effectively flips over as shown in Fig. 2. At first glance this is very unusual as normally the potential is expected to follow a gradual and uniform change throughout the entire volume resulting in the mid equipotential surface being flat. However, this twist in the potential could be explained by the current flow paths inside the CFC panel. We expect the current to take path of least resistance. In an isotropic material this would mean travelling directly through the 
material between the electrodes. The strong anisotropy of the CFC panel, however, creates a different current flow.

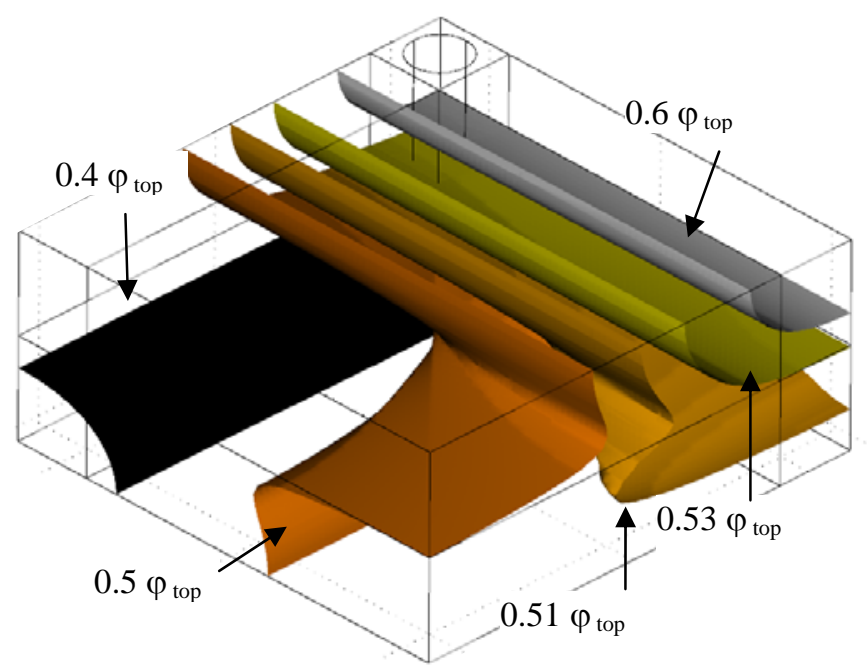

Fig.2. Equipotential surfaces inside the panel. Note the twisting of the $0.5 \mathrm{~V}$ equipotential surface and a small potential drop in the twisting region.

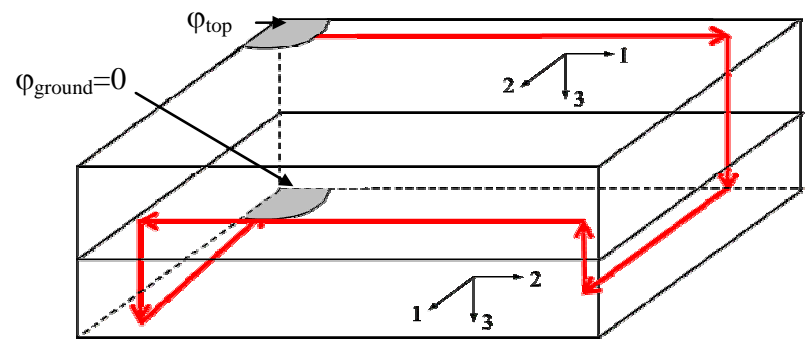

Fig.3. A simplified current flow pattern is shown with the arrows.

Locally the current tends to travel mainly along the local principal axis " 1 " with high $\sigma_{11}$, rather than straight through the material with low $\sigma_{33}$. The diagram in Fig. 3 illustrates the simplified current flow pattern, while Fig. 4 shows the model predictions for the real pattern. Taking the current starting point to be the top electrode, the current initially flows along the principal axis " 1 " in the top layer. As it travels along the top layer, it begins also to penetrate through the thickness of the panel towards the bottom layer. In the bottom layer the direction with high $\sigma_{11}$ is rotated by $90^{\circ}$ in the plane, and so the current prefers to flow in this direction as it reaches the bottom layer, Fig. 3. After having been distributed over a large surface area, the current starts to flow back up into to the top layer, towards the corner far from the electrode in Figs. 3,4. When it re-enters the top layer, it again travels along the top layer's high conductive direction and at some point begins to penetrate back down through the panel into the bottom layer. It can now flow along the high conductive axis to reach the bottom grounded electrode. In summary, the flow path is like travelling around all four sides of the square panel. The interesting observation is that the current travels a much longer distance than expected and also passes the low conductivity directions 3 times.

The rationale behind these somewhat unusual current flows may be better explained by the minimum energy formulation
(3), rather than from (1)-(2), as current density $\mathbf{J}$ is minimised throughout the sample by following the high conductivity directions. Such a distribution abnormally reduces the current density without experiencing significant resistance. The only problem is to connect the currents in the top and the bottom layers, the latter being rotated by $90^{\circ}$. This happens through the twisting in the potential which encounters very small variation along the sides and does not contribute to the Joule heat (3) since the field $\mathbf{E}$ is kept at a minimum value!

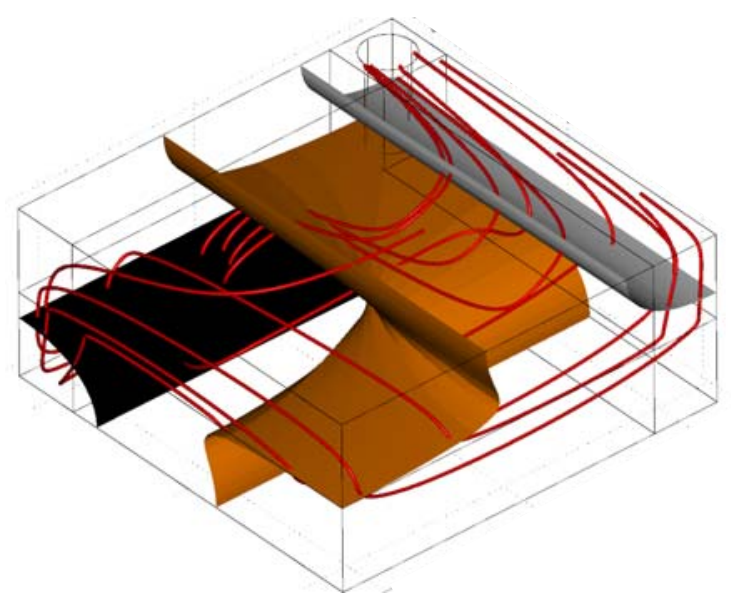

Fig.4. Model predictions for the current flow. The current predominately flows in the direction of fibres, i.e. local " 1 " direction with high conductivity.

\section{DISCUSSION}

The current seeks to minimise Joule heat release via reducing the current density rather than taking the shortest path. This may appear strange as the current travels back and forth through the thickness of the panel three times rather than just once, but it is consistent with the shape of equipotential surfaces. Further investigations will be undertaken to experimentally verify these results.

\section{ACKNOWLEDGEMENTS}

This work has been partially supported by EADS Innovation Works UK, through an industrial studentship.

\section{REFERENCES}

[1] "International encyclopedia of composites ", Ed. S.M. Lee, New York: VCH, 1990.

[2] R.H. Knibbs, J.B. Morris, "The effects of fibre orientation on the physical properties of composites" Composites, vol.5, pp.209-218, 1974.

[3] R. Rolfes, U. Hammerschmidt, "Transverse thermal conductivity of CFRP laminates: A numerical and experimental validation of approximation formulae", Comp. Sci. Techn. vol. 54, pp. 45-54, 1995.

[4] M.R. Kulkarni, R.P. Brady, "A model of global thermal conductivity in laminated carbon/carbon composites", Comp. Sci. Techn., vol.57, pp.277-285, 1997.

[5] J. H. Greenwood, S. Lebedat, J. Bernasconit, "The anisotropic electrical resistivity of a carbon fibre reinforced plastic disc and its use as a transducer," J. Phys. E: Sci. Instr., vol.8, pp.369-370, 1975.

[6] A. Todoroki, M. Tanaka, Y. Shimamura, "Measurement of orthotropic electric conductance of CFRP laminates and analysis of the effect on delamination monitoring with an electric resistance change method," Comp. Sci. Techn., vol.62, pp.619-628, 2002.

[7] COMSOL Inc., "COMSOL Multiphysics simulation environment", Stockholm, Sweden. 